\title{
Medidor de parámetros eléctricos con visualización remota
}

\section{Meter of parameters electric with visualization remote}

MORA-MARTINEZ, Anmar Samael $\dagger^{*}$, VILLALVAZO-LAUREANO, Efrain, JIMÉNEZBETANCOURT, Ramón Octavio y GONZÁLEZ-LÓPEZ, Juan Miguel

\author{
Universidad de Colima \\ ID $1^{\mathrm{er}}$ Autor: Anmar Samael, Mora-Martinez \\ ID $1^{\mathrm{er}}$ Coautor: Efrain, Villalvazo-Laureano \\ ID $2^{\text {do }}$ Coautor: Ramón Octavio, Jiménez-Betancourt \\ ID $3^{\text {er }}$ Coautor: Juan Miguel, González-López
}

DOI: $10.35429 /$ JEA.2020.22.7.23.28

Recibido: Enero 09, 2020; Aceptado: Marzo 25, 2020

\section{Resumen}

En el presente artículo se presenta un medidor de parámetros eléctricos que mide voltaje, corriente y resistencia; todos los parámetros en diferentes rangos de las variables físicas. Con una comunicación remota, por medio de conexión bluetooth para la visualización de los datos en una aplicación diseñada para ser empleada; ya sea, en un dispositivo móvil o un equipo de cómputo. Esta aplicación cuenta con un soporte de una base datos para el almacenamiento de la información que se considere importante; que a su vez puede realizar la función de guardar nuevos datos, actualizar y borrar. Para la creación de la aplicación se utilizó el programa APP INVENTOR y fue enlazada a través de Arduino.

Medidor, Parámetros electricos, APP INVENTOR, ARDUINO

\begin{abstract}
This article presents an electrical parameter meter that measures voltage, current, and resistance, all parameters in different ranges of physical variables. With a remote communication, using a Bluetooth connection for the visualization of the data in an application designed to be used, either on a mobile device or a computer. This application has database support for the storage of information that is considered important, which in turn can perform the function of saving new data, updating and deleting. To create the application, the APP INVENTOR program was used and was linked through Arduino.
\end{abstract}

Meter, Parameters electric, APP INVENTOR, ARDUINO

Citación: MORA-MARTINEZ, Anmar Samael, VILLALVAZO-LAUREANO, Efrain, JIMÉNEZ-BETANCOURT, Ramón Octavio y GONZÁLEZ-LÓPEZ, Juan Miguel. Medidor de parámetros eléctricos con visualización remota. Revista de Aplicaciones de la Ingeniería. 2020. 7-22: 23-28.

\footnotetext{
* Correspondencia del Autor (Correo Electrónico: amora11@ucol.mx)

$\dagger$ Investigador contribuyendo como primer autor.
} 


\section{Introducción}

En el año pasado se realizó un trabajo para la medición de algunos parámetros como son el nivel y la presión; pero además se emplearon para el control en sistema de control de nivel y flujo de agua por medio de la presión diferencial (Rogger, 2019).

En un trabajo se desarrolló un medidor de electricidad de CC para la pila de carga de vehículos eléctricos. En el cual se introducen los parámetros de rendimiento empleando el principio de funcionamiento del medidor; además se diseñan los circuitos de software y hardware del medidor. El instrumento se compone principalmente de las unidades de potencia, muestreo, medición de energía, procesamiento central, visualización, salida y comunicación. A través del muestreo en tiempo real (Lei Song, 2019).

Un artículo presenta un sistema de prueba que se utiliza para la medición de laboratorio y pruebas de parámetros eléctricos. Aunque el sistema fue creado para pruebas de medidores inteligentes, se puede utilizar para pruebas de otros dispositivos (Peter Janiga, 2015).

En otro trabajo se desarrolló un sistema de calibración automática para un multímetro digital; se realizó modificando el calibrador multifuncional tradicional, diseñando el sistema basado en el controlador proporciona integral derivativo y el programa de aplicación (Feng Sha, 2019).

Unos investigadores presentan un método novedoso, de un medidor de potencia que realiza las mediciones por medio de un sistema de visión a través de la computadora en forma automática (Shutao Zhao, 2005).

Desde hace varias décadas en una patente se diseñó un medidor de electricidad de estado sólido, que incluye una unidad de medición electrónica con un convertidor analógico/digital para recibir señales de amplificadores en diversos rangos de medición, y una sección de control con un microcontrolador en la que se procesa la señal del medidor (United States Patente no 4,837,504 , 1989).
En la misma década se patentó un medidor de energía eléctrica para medir diversos parámetros de consumo de energía eléctrica durante períodos de tarifa de facturación diferenciados (United States Patente $\left.\mathrm{n}^{\circ} 4,511,979,1985\right)$.

Otro diseñador patentó un medidor que emplea un procesador que tiene una memoria asociada para recibir una entrada de voltaje analógico y señales de corriente, para procesar estas señales con el propósito de generar datos representativos de las mediciones de electricidad e información relacionada (al H. e., 1996).

En una investigación se propone un enchufe especial para un medidor de vatioshora; es un conector al medidor inteligente que no necesita cable al circuito móvil. Es un nuevo método de conexión e instalación al medidor inteligente y consta de un tapón metálico y soporte aislante (Zhang Zhi, 2017).

\section{Módulo de medición de las variables}

El módulo de medición de las variables cuenta con tres formas de medición que son, voltaje, resistencia y corriente. Para lograr esta función se utilizó un ARDUINO MEGA 2560 para leer los datos y mostrarlos en forma local en una LCD (16x2), los datos recibidos se convirtieron de analógico a digital con los pines ANALOG IN del Arduino; cómo se puede observar en la Figura 1:

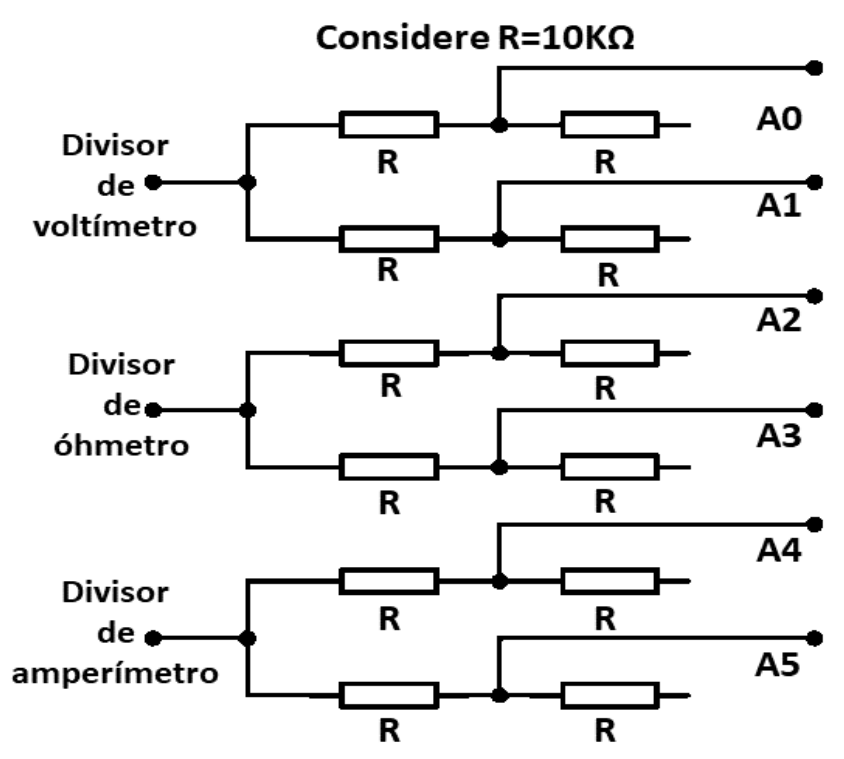

Figura 1 Conexión de los pines analógicos 
Se usaron divisores de corriente y voltaje para llevar los niveles de las variables medidas a parámetros soportables por el Arduino; el nivel máximo de voltaje es de $5 \mathrm{~V}$ y con ello se logró tener varias escalas disponibles. En el caso de las resistencias se manejó un divisor de tensión, sólo para poder leer la segunda resistencia que es el valor que se desea medir; cómo se puede observar en la Figura 2:

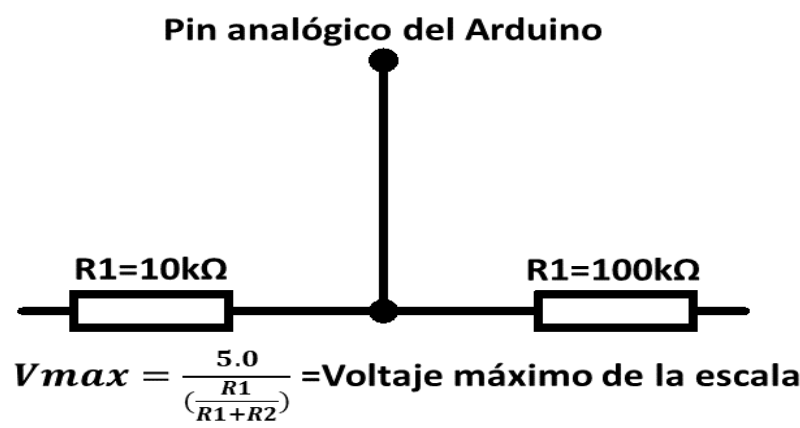

Figura 2 Divisor de voltaje y cálculo para la escala

En el programa ARDUNIO se establecieron los cálculos; para mostrar las mediciones en la LCD y enviar una alerta en caso de sobrepasar el voltaje o corriente permitidos por el instrumento, se realiza por medio de las ecuaciones 1 y 2 .

$$
\begin{aligned}
& V_{\text {out }}=((\text { value } * 4.8)+20) / 1024.0 \\
& V_{\text {in }}=\left(V_{\text {out }} /\left(R_{1}+R_{2}\right)\right)
\end{aligned}
$$

Para el cambio de escalas se empleó el comando IF y tres interruptores para indicar las posiciones de los interruptores según la escala o medidor como se muestra en las siguientes líneas de código, de forma similar se realiza para cada una de las escalas.

*********************************************

Asignación de valores a las variables (para este caso voltaje)

******************************************** if $(V==L O W \& \& R==L O W \& \& A==L O W) / /$ Elección de escala

*******************************************

Asignación de valores a las variables

******************************************* int analogInput $=0$;

float vout $=0.0$

float vin $=0.0$;

float $R 1=22000$;

float $R 2=9780.0$;

int value $=0$;

float val $=0$;

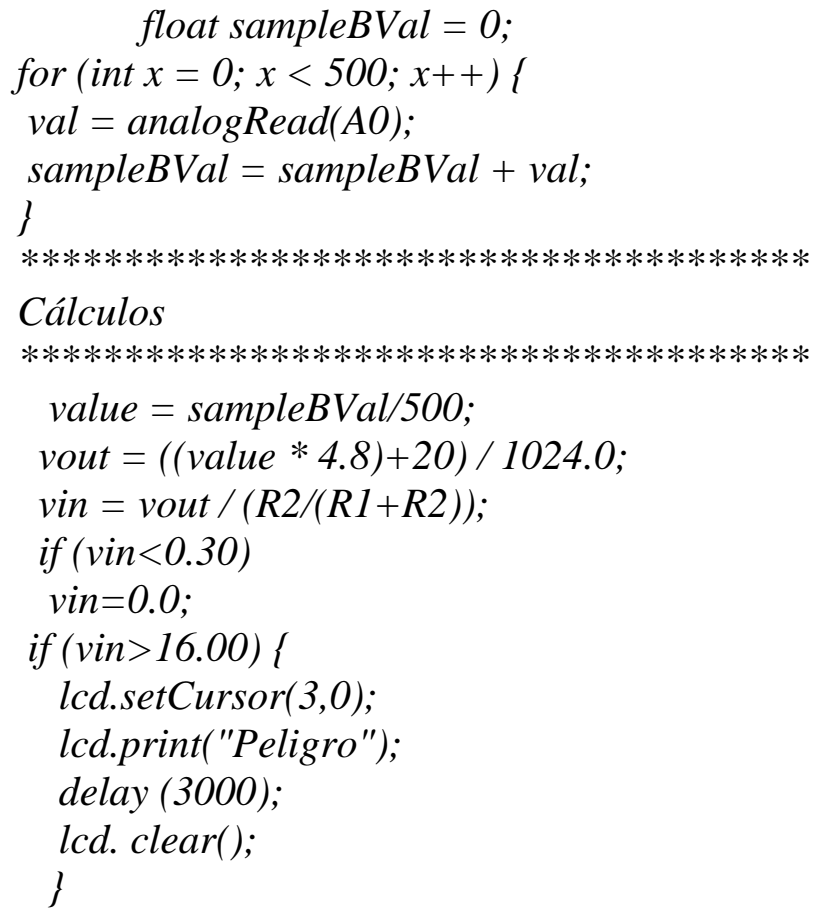

Para la conexión bluetooth se recurrió a un módulo Bluetooth HC-C6. Este módulo se conecta al Arduino a las terminales TX y RX; estos puertos permiten mandar y recibir datos, el dispositivo según la carta de datos requiere de $5 \mathrm{v}$ como para poder estar en funcionamiento, las conexiones se muestran en la figura 3 .

El medidor de parámetros cuenta con un interruptor de encendido y apagado para hacerlo portátil, cuenta con dos baterías en conectadas en paralelo para poder aumentar la corriente de alimentación; puesto que una sola batería no tiene la capacidad suficiente de soportar todos los componentes; el circuito de conexión de las baterías se puede observar en la Figura 4:

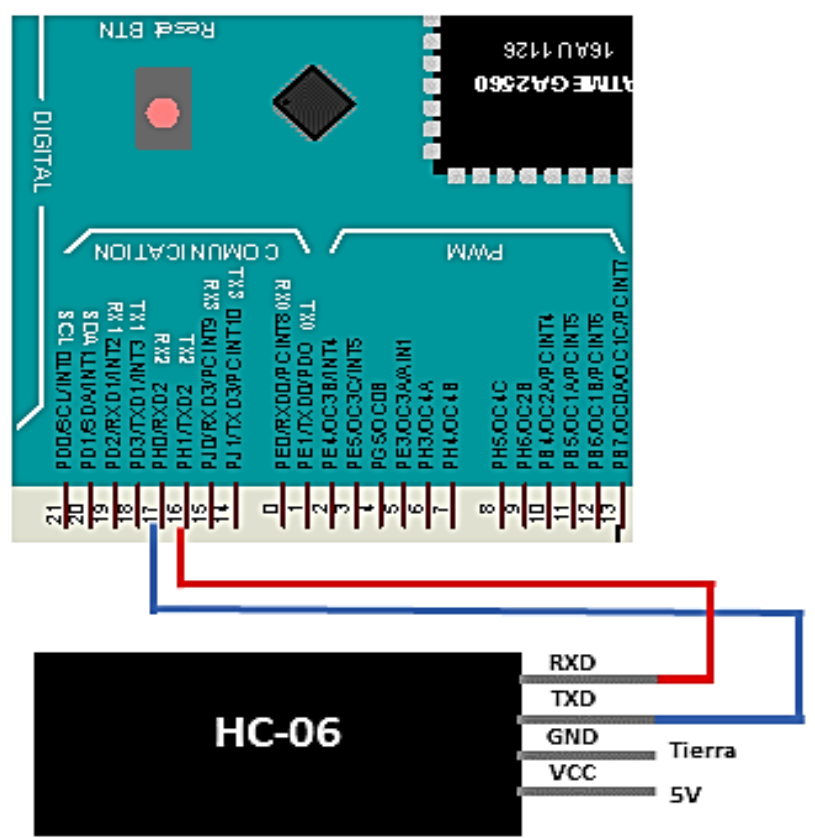

Figura 3 Conexiones Arduino con módulo bluetooth

MORA-MARTINEZ, Anmar Samael, VILLALVAZO-LAUREANO, Efrain, JIMÉNEZ-BETANCOURT, Ramón Octavio y GONZÁLEZLÓPEZ, Juan Miguel. Medidor de parámetros eléctricos con visualización remota. Revista de Aplicaciones de la Ingeniería. 2020 


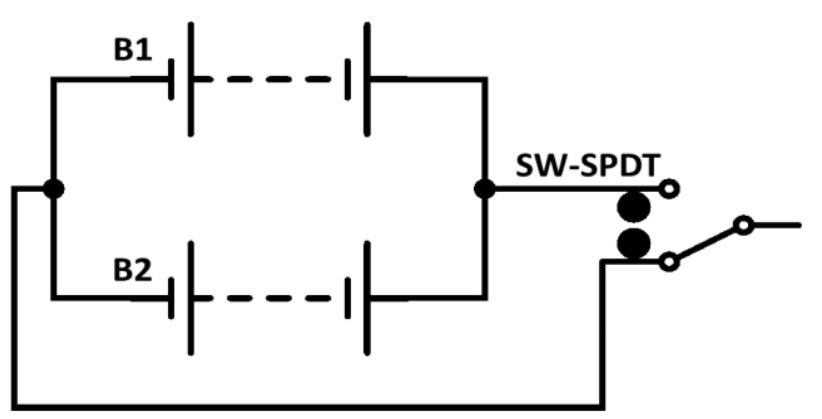

Figura 4 Conexión de las baterías de alimentación en paralelo

La carcasa del multímetro está hecha de acrílico un plástico duro que puede proteger los componentes. En el frente de la carcasa se utilizaron números binarios para poder seleccionar las escalas y orificios de conexión para introducir las puntas de medición. En el programa Arduino se usó el comando "serial.Print()" para mostrar el texto según la posición de los interruptores y el comando "serial.Println()" es para el valor que va a leer y mostrar en la aplicación como se visualiza en las líneas de código siguientes.

Serial.print("15v: "); // Texto que se muestra en el celular, debido al valor de la variable de entrada.

Serial.println(vin); // Valor de la variable de entrada para mostrar en el celular

En la figura 5 se muestra la parte frontal del medidor; con los interruptores para la selección de los parámetros que se desean medir.

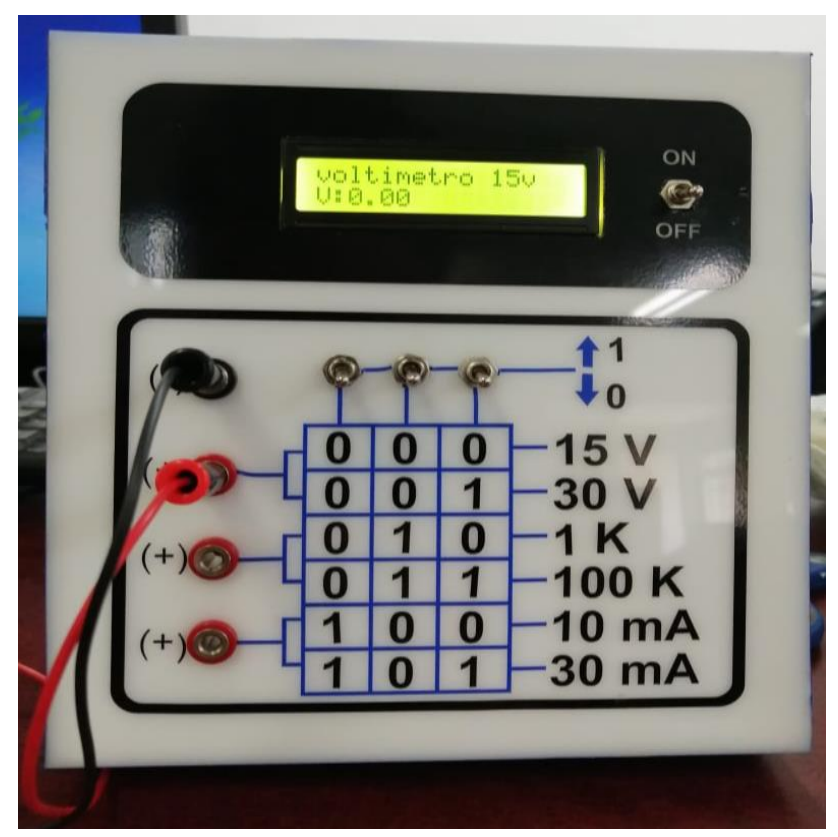

Figura 5 Funcionamiento de los interruptores del medidor de los parámetros eléctricos

\section{Aplicación}

Para la creación de la aplicación se usó APP INVENTOR, en esta aplicación se desarrolló el diseño y el código para poder hacer la conexión bluetooth; así como la base de datos, este programa se creó con bloques para facilitar el desarrollo de las aplicaciones.

Se agregó un comando CLOCK1, que es el tiempo que va a tardar en mostrar los datos enviados hacia la aplicación, se estableció un comando llamado bluetoothclient1 este permite hacer la comunicación del teléfono al Arduino.

Se hace la conectividad del teléfono al módulo bluetooth y después se confirma si el dispositivo está conectado correctamente. Cuando la aplicación recibe bytes mayores a 0 , es cuando muestra el valor o lo que se ha seleccionado con los interruptores.

Una vez que se efectuó la conectividad; en el comando CLOCK1 se establece en que parte se van a mostrar los datos, que en este caso fue en LABEL1 la que muestra los datos mayores a 0 . cómo se puede observar en la Figura 7:

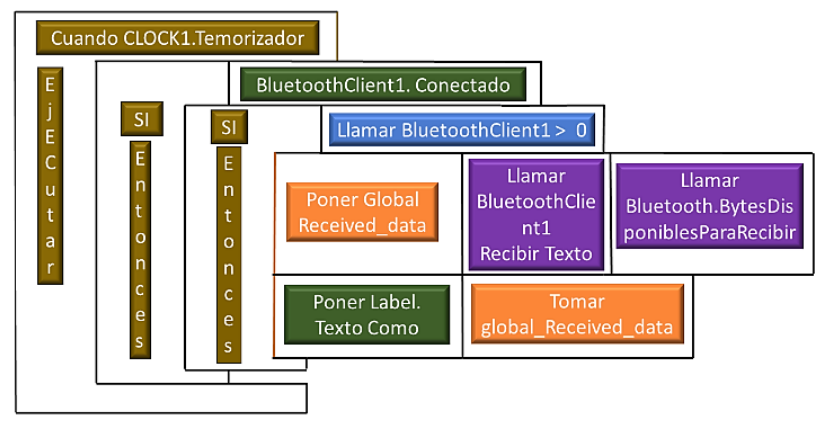

Figura 6 Ejemplifica como fueron colocados los bloques para poder mostrar los datos en la aplicación y verificar la conectividad

La aplicación cuenta con una base de datos simple que también se desarrolló en App Inventor, se usó el comando TINYDB1, este comando guarda los datos en la misma aplicación como se presentan en la figura 7.

Se usaron botones con el propósito de guardar, actualiza o borrar datos; cómo se puede observar en la Figura 8: 


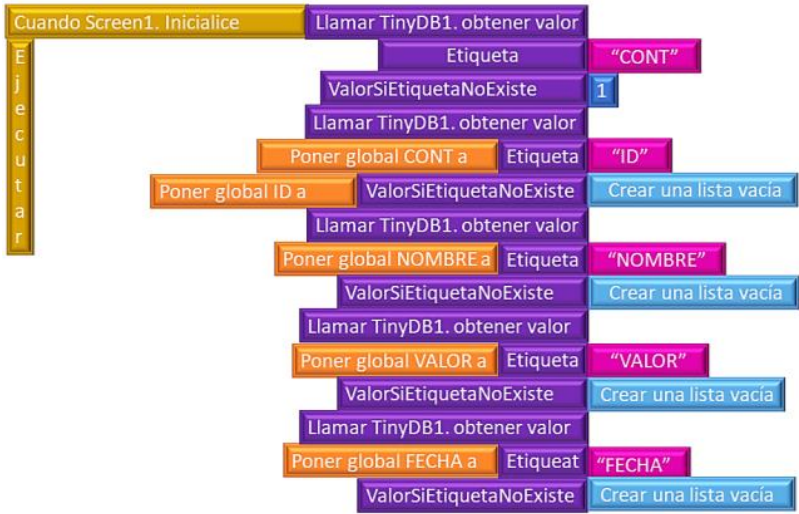

Figura 7 La imagen muestra donde se inicializa y se usa el comando TINYDB1

En el caso del registro; sólo se usó un botón llamado agregar, en actualizar se usaron dos botones que es buscar y actualizar, es lo mismo para el caso de borrar dato como se ejemplifican en la figura 8.

\section{REGISTRO}

\begin{tabular}{l|l|} 
Nombre \\
Valor \\
Fecha
\end{tabular}

Agregar

Figura 8 Muestra cómo se observará el espacio de agregar un dato en la aplicación

\section{Borrar}

$$
\text { ID }
$$

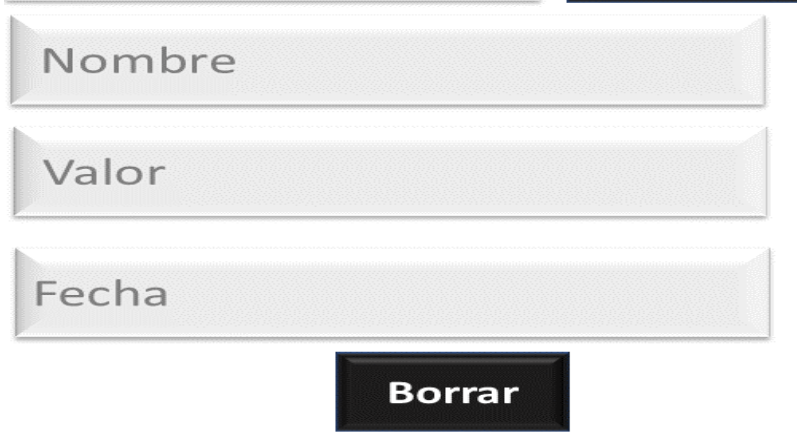

Figura 9 Aquí se muestra cómo será el espacio de borrar en la aplicación, sería lo mismo para el caso de actualizar

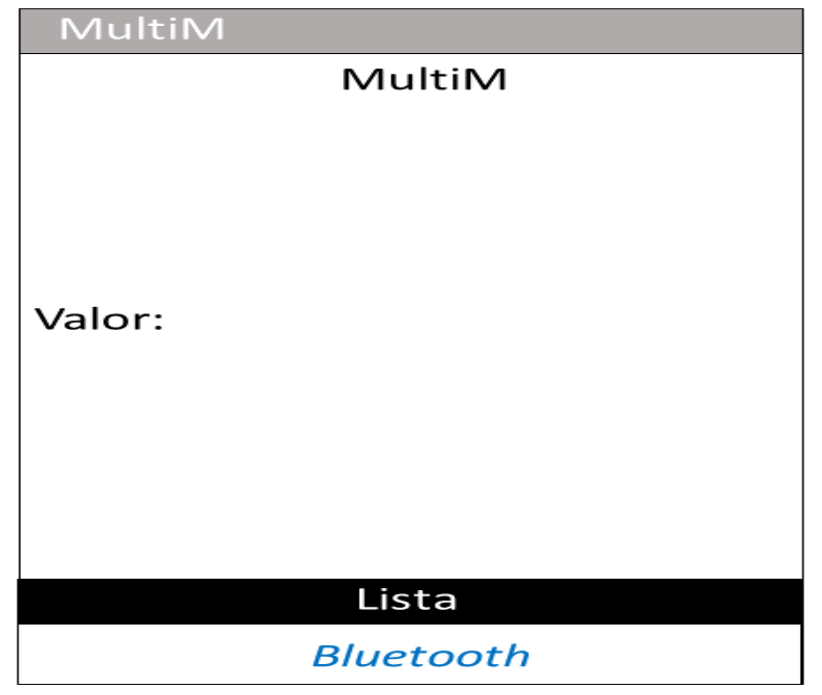

Figura 11 Imagen muestra los comandos usados para el tiempo, la conectividad bluetooth y la base de datos

\section{Agradecimientos}

A la Universidad de Colima por el apoyo en los espacios para la realización de este proyecto y a los estudiantes de Ingeniería en Tecnologías Electrónicas Jesús José María Rebollar, Juan Manuel Villalvazo Guzmán y Jaret Ernesto Albores Chiñas por su colaboración en algunas pruebas físicas.

\section{Conclusiones}

El medidor de parámetros eléctricos es muy útil para la enseñanza en la medición; porque con la configuración física por medio de interruptores es muy intuitivo para principiantes en las mediciones.

El software de desarrollo es una plataforma abierta que permite tener acceso a las modificaciones y actualizaciones sin generación de costo por mantenimiento.

Tiene la ventaja de poder realizar mediciones locales y enviarlas en forma remota a una aplicación para celular.

Cuenta con una base de datos simple, para el almacenamiento de los datos que se consideren importantes.

\section{Trabajos futuros}

- Es conveniente realizar el instrumento para otras variables como potencia y armónicos.

- Agregar registros o tendencias de las variables.

MORA-MARTINEZ, Anmar Samael, VILLALVAZO-LAUREANO, Efrain, JIMÉNEZ-BETANCOURT, Ramón Octavio y GONZÁLEZLÓPEZ, Juan Miguel. Medidor de parámetros eléctricos con visualización remota. Revista de Aplicaciones de la Ingeniería. 2020 


\section{Referencias}

al, B. e. (1989). Patente $n^{o} 4,837,504$. United States .

al, B. e. (1989). Patente $n^{o} 4,837,504$. United States .

al, H. e. (1996). Patente $n^{o} 5,548,527$. United States.

Amirante. (1985). Patente $n^{\circ} 4,511,979$. United States .

Feng Sha, M. L. (2019). The Design of Digital Multimeter Automatic Calibration System and Application of PID Algorithm. 8th Joint International Information Technology and Artificial Intelligence Conference, 1302 to 1305.

Lei Song, Y. X. (2019). Development of a Novel DC Electricity Meter Based on New Electronic Current Transformer. 3rd Information Technology,Networking,Electronic and Automation Control Conference, 1702 to 1702.

Peter Janiga, M. L. (2015). Testing system for smart meters. 16th International Scientific Conference on Electric Power Engineering (EPE), 1 to 4.

Shutao Zhao, B. L. (2005). Research on Remote Meter Automatic Reading Based on Computer Vision . Transmission and Distribution Conference \& Exhibition: Asia and Pacific Dalian, China, 1 to 4.

Zhang Zhi, D. X. (2017). The research of performance testing about watt-hour meter plug. 13th International Conference on Electronic Measurement \& Instruments, 458 to 461. 